\title{
Réveil religieux et sortie de la religion en Chine contemporaine
}

\section{Benoît Vermander}

\section{(2) OpenEdition \\ Journals}

Édition électronique

URL : http://journals.openedition.org/perspectiveschinoises/5352

ISSN : 1996-4609

\section{Éditeur}

Centre d'étude français sur la Chine contemporaine

\section{Édition imprimée}

Date de publication : 31 décembre 2009

ISBN : 978-2-95333678-7-4

ISSN : 1021-9013

Référence électronique

Benoît Vermander, "Réveil religieux et sortie de la religion en Chine contemporaine », Perspectives chinoises [En ligne], 2009/4 | octobre-décembre 2009, mis en ligne le 01 décembre 2012, consulté le 19 avril 2019. URL : http://journals.openedition.org/perspectiveschinoises/5352 


\title{
Réveil religieux et sortie de
}

\section{la religion en Chine}

contemporaine

\author{
BENOÎT VERMANDER
}

Cet article étudie à la fois le renouveau d'organisations et de pratiques religieuses en Chine et ce que l'on pourrait appeler une " sortie de la religion " caractérisée par la perte de tout socle religieux soudant le lien social et par une instrumentalisation des pratiques et des discours religieux. Il défend l'idée suivant laquelle la prise en compte de ce double phénomène de " renouveau " et de " sortie " facilite non seulement notre compréhension de la sphère religieuse et de ses évolutions, mais aussi celle de la configuration sociopolitique dans laquelle le pays est engagé.

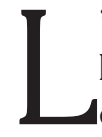

'étude des religions en contexte chinois se heurte dès l'abord à des difficultés de principe : le terme même religion ", emprunte des langues occidentales par l'intermédiaire du japonais, est-il pertinent pour définir les formes sociales étudiées ${ }^{(1)}$ ? Et si l'on s'autorise à parler d'une "sphère religieuse ", les concepts occidentaux fournissent-ils autre chose que des analogies souvent trompeuses pour en décrire les structurations mentales et sociales ${ }^{(2)}$, même lorsqu'ils sont spécifiquement aménagés pour rendre compte des réalités chinoises ${ }^{(3)}$ ? Mais alors quel vocabulaire et quelles catégories employer pour rendre compte de l'ordre des phénomènes ordinairement étudiés par les " sciences religieuses" occidentales ? À l'inverse, une concentration trop étroite sur les problèmes lexicographiques ne dissimule-t-elle pas ce qu'ont d'universel les expériences et manifestations religieuses chinoises, interdisant du coup l'entrée dans une démarche comparatiste ? Enfin, dans quelle mesure la détermination par l'État de formes religieuses authentiques et " acceptables » oriente ou déforme-t-elle notre perception du fait religieux chinois dans la spontanéité de ses manifestations sociales? Ces questions, qui peuvent être enrichies et multipliées, montrent les difficultés de principe rencontrées par toute étude portant sur " les religions en Chine ».

\section{Les religions en Chine: démographie et ethnographie}

\section{Un cadre d'exercice}

Rappelons tout d'abord que, depuis 1949 et jusqu'à présent, cinq formes religieuses seulement sont légalement reconnues, que chacune de ces religions est " encadrée " par une association qui fait office de courroie de transmission entre cette expression donnée de la société civile et l'État-Parti dont elle transmet les orientations et consignes ${ }^{(4)}$, et que, par ailleurs, l'Administration d'État des affaires religieuses ${ }^{(5)}$, aux différents échelons territoriaux, fonctionne comme une sorte de " ministère des Cultes " aux compétences très étendues. Enfin, dès les débuts du nouveau régime, les " cultes hétérodoxes " et les "superstitions ", distingués des "religions ", ont été formellement bannis, et les premiers ont fait parfois l'objet d'une répression systématique, illustrée dès 1951 par la violente campagne menée contre le mouvement Yiguandao. Ce rappel préliminaire suggère déjà des schèmes de compréhension. En effet, le système ainsi mis

1. Joël Thoraval, «Pourquoi les "religions chinoises" ne peuvent-elles apparaître dans les statistiques occidentales ? ", Perspectives chinoises, $\mathrm{n}^{\circ} 1$, mars 1992, p. 37-44; Vincent Goossaert, «1898: The Beginning of the End for Chinese Religion? ", Journal of Asian Studies, 65(2), 2006, p.307-336.

2. Liu Xiaogan, cité par Fan Lizhu, qualifie d' « interprétation analogique inversée » la pratique des chercheurs chinois d'appliquer un concept occidental à la compréhension de leur propre univers de croyance, travaillant ainsi à l'inverse des philosophies chinois des premiers siècles de notre ère qui faisaient usage de concepts familiers tels celui de Dao pour rendre compte de notions bouddhistes importées. Cf. Fan Lizhu, «The Dilemma of Pursuing Chinese Religious Studies in the Framework of Western Religious Theories ", Fudan Journal of the Humanities and Social Sciences, 2(2), Juin 2009, p.29-48, notamment p.30.

3. Fan Lizhu note que le célèbre concept de diffused religion articulé par C.K. Yang pour rendre compte de la spécificité des formes sociales du religieux en Chine a été traduit en chinois parfois avec la connotation de « dispersée » (sankaide), de "élargie » (kuosanxing), de "répandue " (misanxing) ou de " distribuée " (fensanxing) (Fan, "The Dilemma of Pursuing Chinese Religious Studies in the Framework of Western Religious Theories ", art. cit., p. 38)

4. Le «Comité patriotique protestant des trois autonomies » fut fondé en 1954, l'Association bouddhiste de Chine en 1955, les associations islamiste, taoïste et catholique en 1957. Les structures peuvent être plus complexes que ne l'indique ce rapide résumé. À strictement parler, l'Église protestante est formée par l'association du «Conseil chrétien chinois » (fondé en 1980) et du « Comité patriotique » mentionné à l'instant. Le rapport entretenu entre l'association patriotique catholique et la conférence épiscopale des évêques " officiels » est de même nature.

5. Auparavant appelé Bureau des affaires religieuses. 
en place instaure une double coupure, tout en maintenant la possibilité de sauvegarder un " continuum " de la sphère religieuse : coupure entre formes religieuses reconnues et non reconnues (quel que soit le degré d'institutionnalisation de ces dernières) ; et, à l'intérieur même des religions reconnues, coupure entre les groupes et personnalités participant aux structures mises en place par l'État-Parti et ceux qui s'y soustraient. Pourtant, l'observation amène à parler moins de coupure que d'un " continuum » déterminé par la variété de stratégies des différents acteurs quant à leur degré d'inclusion dans l'appareil officiel (de l'adhésion totale à la dissidence, en passant par tous les intermédiaires).

L'article 36 de la Constitution de la $\mathrm{RPC}^{(6)}$ stipule que:

les citoyens de la République populaire de Chine disposent de la liberté de croyance religieuse. Aucun organe d'État, organisation publique ou individu ne peut contraindre des citoyens à croire, ni discriminer les citoyens croyants ou non-croyants. L'État protège les activités religieuses normales. Personne ne peut se servir de la religion pour engager des activités qui perturbent l'ordre public, ni altérer la santé des citoyens ni interférer dans le système d'éducation de l'État. Les corps religieux et les affaires religieuses ne sont sujets à aucune domination étrangère.

Contrôle public, liberté individuelle de croyance, détermination par l'État de la « normalité » des croyances et de leurs manifestations, cadre national strictement assigné au religieux - tels sont donc les principes énoncés.

Abstraction faite de la période 1966-1979, la continuité du cadre légal et idéologique mis en place par le régime pour la gestion des religions l'emporte sur les évolutions. Le cadre légal actuel est déterminé en 1982, mais les principes qu'il énonce étaient mis en action dès le tout début des années 1950 (une exception notable est l'affirmation que la disparition des religions est un objectif de long terme, affirmation énoncée en 1982). Une évolution sourde s'esquisse au tournant du siècle : la lutte contre les " cultes pervers ", essentiellement, à partir de 1999, contre Falungong, a renforcé les positions des formes religieuses sanctionnées par l'État. Il s'agit d'une évolution et non d'une rupture dans la mesure où les religions ont été fonctionnalisées par le gouvernement chinois au fur et à mesure du développement de la politique de réforme et d'ouverture, au titre de pourvoyeuses subordonnées de moralité, d'encadrement social et d'ouverture internationale. Par ailleurs, à la reconnaissance du rôle social positif que peuvent jouer les religions s'ajoute désormais une reconnaissance prudente de la différence entre les «cultes pervers " (xiejiao) (désormais assimilés aux " sectes " du monde occidental) et les " nouveaux mouvements religieux " (xinxing zongjiao), ouvrant peut-être ainsi la voie à un assouplissement progressif des catégories régissant le champ religieux ${ }^{(7)}$, assouplissement implicitement suggéré par les Régulations sur les affaires religieuses de mars 2005.

Enfin, la nature même du cadre institutionnel fourni par les associations-relais renvoie à l'histoire des 100 dernières années, laquelle a connu la constitution progressive de religions " nationales " et " modernes " : des associations religieuses nationales voient le jour dès 1912 et son cooptées par l'État au cours des années $1930^{(8)}$, même si elles sont reformatées en fonction des besoins du nouveau régime à partir de 1949. La rupture de 1949 ne doit donc pas dissimuler la constitution d'une sphère " moderne » du religieux à partir de la fin du XIX siècle, mais il faudra nous demander s'il est suffisant de reporter la rupture instauratrice à une date antérieure à 1949 (aux alentours de $1898^{(9)}$ ) pour rendre compte de la dynamique du phénomène religieux chinois.

\section{Compter les croyants...}

Le contrôle étatique des expressions religieuses ne va pas de pair avec la collecte de données statistiques. Les recensements ne comprennent pas de questions sur l'appartenance religieuse. L'Institut chinois de sondages d'opinion Horizon Group (Lingdian jituan) a réalisé deux enquêtes en collaboration avec "Pew Global Attitudes Project " en 2005 et 2006, et une autre enquête en 2007 avec C100, une organisation non partisane de citoyens américains d'origine chinoise ${ }^{(10)}$. La base d'enquête surreprésente le milieu

6. Rendu public en mars 1982 , le « document 19 » du Comité central avait déjà formulé les principes qui servent de base à l'article 36 .

7. Cf. David Palmer, « Heretical Doctrines, Reactionary Secret Societies, Evil Cults: Labeling Heterodoxy in Twentieth-Century China ", in Mayfair Mei-hui Yang (éd.), Chinese Religiosities, Afflictions of Modernity and State Formation, Berkeley, University of California Press, 2008, notamment p.129-134.

8. Constitution de deux associations bouddhistes concurrentes vers 1912, l'une d'orientation plus laïque et l'autre plus monastique; situation à peu près similaire pour la structuration nationale du taoïsme ; création d'une association islamique nationale; floraison des tentatives d'organisation nationale du confucianisme; structuration nationale plus tardive des églises chrétiennes, du fait des divisions confessionnelles, des influences étrangères et du poids des associations spécialisées déjà existantes. Cf. Vincent Goossaert, « Republican Church Engineering: The National Religious Associations in 1912 China », in Mayfair Mei-hui Yang (éd.), Chinese Religiosities, Afflictions of Modernity and State Formation, Berkeley, University of California Press, 2008, p. 209-232.

9. Cf. Vincent Gossaert, "L'invention des "religions" en Chine moderne », in Anne Cheng (dir.), La pensée en Chine aujourd'hui, Paris, Gallimard, col. "Folio Essais », 2007, p. 185-213 ; du même : «1898: The Beginning of the End for Chinese Religion? », Journal of Asian Studies, 65(2), 2006, p. 307-336.

10. Synthèse des trois enquêtes disponible sur pewforum.org, 2 mai 2008, consulté le 30 juillet 2009. Analyse effectuée par Brian J. Grim, Pew Forum on Religion \& Public Life. 
urbain $^{(11)}$ et est donc représentative d'environ la moitié de la population adulte de la Chine. Six villes et leurs environs immédiats ont été sondés en 2005 et 2006, et sept en 2007. L'enquête de 2006 donne les indications suivantes : $31 \%$ des interrogés considèrent la religion comme étant " très ou assez importante dans (leur) vie ", $11 \%$ affirmant qu'elle n'est pour eux "pas du tout importante ». L'enquête de 2005, avec une formulation quelque peu différente de la question, donnait un pourcentage plus élevé encore (56\%) de personnes considérant la religion comme très ou assez importante dans leur vie.

Dans ces trois enquêtes, entre $14 \%$ et $18 \%$ des personnes interrogées ont revendiqué une appartenance religieuse. À titre de comparaison, une autre étude menée par des chercheurs affiliés avec l'Université normale de la Chine de l'Est (Shanghai), et disponible sous la forme de résultats publiés dans China Daily ${ }^{(12)}$, indiquait que " $31,4 \%$ des Chinois de 16 ans et plus, ou environ 300 millions de personnes, sont religieux. " L'intérêt de cette dernière enquête ne réside pas tant dans le chiffre annoncé que dans le fait que, pour la première fois, un média sous contrôle de l'État ne citait pas le chiffre habituel de " 100 millions de personnes professant une foi religieuse ${ }^{(13)}$ ").

Dans les trois études effectuées par Horizon Group, les bouddhistes forment le premier groupe religieux en Chine, entre $11 \%$ et $16 \%$ de la population adulte. Ces études confirment aussi la répugnance de la population chinoise à s'identifier comme " taoiste ", puisque cette appartenance est revendiquée par moins de $1 \%$ des sondés. Et elles indiquent que moins de $4 \%$ des sondés se revendiquent chrétiens. Les réticences associées à la revendication de cette appartenance dans le cas de sondés n'appartenant pas à des groupes ou églises officiellement reconnus permettent de supposer que ce chiffre minore le pourcentage effectif (l'effet de la surreprésentation urbaine de ces sondages est plus difficile à estimer). Les questions du sondage de 2005 en incluaient certaines sur la " croyance personnelle » dans " la fortune (yun)» (40\% de réponses affirmatives) ${ }^{(14)}$, "Bouddha » $(8 \%)$ et « le Dieu Jésus (shangdi Yesu) " (6\%). Ces réponses ne sont pas en contradiction flagrante avec celles portant sur les appartenances : une revendication plus élevée d'appartenance bouddhiste peut correspondre par exemple à une appréciation positive du système de moralité qui est attaché au bouddhisme, sans croyance affirmée à une figure particulière ${ }^{(15)}$, tandis que la croyance en la figure de "Jésus " peut aller de pair avec l'absence d'appartenance déclarée à une église. Le recoupement des enquêtes et la prise en considération de la marge d'erreur statistique permettent de penser que, pour la population urbaine au moins, les estimations ainsi proposées ne sont pas infondées. Les mêmes enquêtes livrent le chiffre de près de $1 \%$ de la population adulte déclarant son appartenance à la religion musulmane, probablement une forte sous-estimation liée aux limites géographiques des enquêtes. Une estimation de l'appartenance à l'Islam basée sur les données ethniques livre un chiffre compris entre 20 et 23 millions de personnes, encore que cette base d'estimation soit bien entendu elle aussi sujette à critique.

Une enquête parallèle aux précédentes et menée en avril et mai $2005^{(16)}$ ne décèle pas de différence dans l'intérêt manifesté envers la religion par les hommes et les femmes, ni de variation significative en fonction des groupes d'âge ${ }^{(17)}$. Elle constate aussi un intérêt plus élevé chez les citadins (24\%) que parmi la population rurale (18\%) (mais l'enquête est probablement limitée à la périphérie immédiate des villes et aux ruraux vivant en milieu urbain). Les titulaires d'un diplôme universitaire expriment un intérêt religieux plus marqué (26\%) que ce n'est le cas chez les détenteurs d'un diplôme d'un collège d'enseignement professionnel (18\%). Les fonctionnaires de l'État et du Parti expriment l'intérêt le plus fort (33\%). En termes de catégories de revenu, l'intérêt pour le sujet est presque identique dans tous les groupes, sauf pour ceux qui n'ont pas de revenu ou aucun revenu fixe $(15 \%)$

11. Les études sur l'évolution présente des activités religieuses dans les campagnes chinoises sont plus rares, et les difficultés rencontrées dans l'étude de terrain rendent leurs conclusions prudentes. Par ailleurs, les chercheurs chinois se débattent avec la signification de la « fièvre religieuse » des campagnes, fièvre que la plupart s'accordent pourtant à détecter. Les explications avancées incluent : une liaison organique de la croissance économique et de la croissance religieuse ; le poids croissant des « quatre groupes nombreux » (les vieux, les femmes les non éduqués, les malades et handicapés...) plus susceptibles de s'en remettre aux croyances religieuses ; la liaison entre influence culturelle de l'Occident et fièvre religieuse l'activité plus grande des religions dans la sphère profane ; la confusion entre religions et superstitions populaires en progrès. Cf. notamment Dong Jianbo, Li Xuechang, « Zhongguo nongcun zongjiao xinyang de bianqian » (Les transformations de la croyance religieuse dans les campagnes chinoises), Shanghai Xinzhengyuan xuebao (Journal of Shanghai Administrative Institute), septembre 2004, 5 (5), notamment p. 102-103.

12. 7 février 2007, chinadaily.com.cn, dernière consultation le 30 juillet 2009.

13. II est vrai que ce chiffre, souvent répété depuis la fin des années 1990, ne correspondait plus à grand chose, puisque Xinhua affirmait par exemple le 12 avril 2006 que l'on compte environ 100 millions de bouddhistes en Chine (xinhuanet.com.english, consulté le 30 juillet 2009).

14. II est intéressant de noter qu'avec un taux $40 \%$, la croyance dans la chance ou la fortune (yun), réalité spirituelle "manipulable », est plus forte que celle dans le destin (ming) (29\%), dont on ne peut maîtriser le cours.

15. Les enquêtes menées à Taiwan ont souvent montré la revendication d'une appartenance bouddhiste avec faible corrélation des réponses portant sur les croyances et les pratiques. Synthèse in Benoît Vermander, « Le paysage religieux de Taiwan et ses évolutions récentes ", L'Ethnographie, 1995, 118, p. 9-59.

16. Enquête menée par le groupe InterMedia. Données achetées et analysées par Pew, cf pewforum.org, 2 mai 2008, consulté pour la dernière fois le 30 juillet 2009. La question posée est sujette à multiples interprétations : "Êtes-vous intéressé par la religion et par le fait que le sujet soit abordé dans les médias? » Une réponse positive ne présuppose donc ni croyance ni appartenance religieuse.

17. En contraste, l'enquête de l'Université normale de la Chine de l'Est citée dans l'article du China Daily du 7 février 2007 relève que les deux tiers des personnes qui se déclarent « religieuses » sont âgées de 16 à 39 ans. 

y dirige ses vœux et ses prières, notamment pour les défunts. Lieu de savoir, les grands temples permettent de poursuivre sur plusieurs siècles la traduction du canon bouddhique en chinois et d'en multiplier les interprétations. Lieu de pouvoir, le temple sait négocier ses relations avec les Grands de la localité puis de l'Empire, encore que ce modèle soit mis en échec lors de la grande persécution du IX $\mathrm{X}^{\mathrm{e}}$ siècle, causée en partie par la concentration de richesses accomplie par les communautés monastiques.

\section{Le bouddhisme chinois est ainsi devenu un puissant} pouvoir religieux qui tirait sa force de cette remarquable institution qu' est le monastère. Mais le fait surprenant est que cette grande puissance religieuse se soit constituée sans aucune forme de direction ou de coordination centrale. Le bouddhisme chinois a toujours été un océan d'innombrables centres, grands et petits, de niveaux très différents, les plus grands patronnés par la cour et peuplés de moines cultivés, les plus petits végétant dans les villages et occupés par quelques moines illettrés. En résumé : une grande force institutionnelle combinée à une grande faiblesse d'organisation ${ }^{(18)}$.

La reconstruction du bouddhisme chinois après la tourmente de la Révolution culturelle s'est donc appuyée sur l'institution monastique, comme cela avait déjà été le cas à d'autres époques. Et la vitalité des monastères témoigne de celle des pratiques et croyances bouddhistes dans l'ensemble de la société. De façon intéressante, c'est sans doute la seconde partie du constat d'Erik Zürcher, sur la faiblesse organisationnelle du réseau bouddhiste, qui s'avère moins pertinente pour la période actuelle, et cela du fait des caractéristiques propres à la structure du Parti-État. L'affirmation du rôle de l'Association bouddhiste chinoise et la création concomitante de " courroies de transmission » entre le pouvoir et les organisations religieuses locales vont de pair avec une communication et une solidarité plus grandes entre les divers centres, grands ou petits qui, pris en leur ensemble innervent le bouddhisme chinois. En d'autres termes, le bouddhisme chinois forme peut-être aujourd'hui un tout plus robuste et plus solidaire quil ne le fut jamais dans le passé.

La nature et l'usage du pouvoir attaché aux monastères, tel qu'il est aujourd'hui exercé par une élite de jeunes clercs immergés dans cette tâche de reconstruction et d'expansion, dépendent pour bonne part de la transformation intervenue dans les bases économiques des monastères : l'exploitation des domaines agricoles a été remplacée par une dépendance accrue envers les dons (de l'étranger d'abord, puis en provenance de donateurs locaux), l'aide des agences gouvernementales (pour la reconstruction des bâtiments notamment), la pratique des rituels, le tourisme et les activités charitables. Les moines affiliés à un monastère donné en reçoivent en général une modeste allocation qu ils complètent grâce à leurs talents liturgiques ou par d'autres moyens. Dans les monastères tibétains pour le moins, les bénéfices d'une allocation et d'un logement au monastère sont subordonnés à l'exercice d'un service rendu à la communauté monastique (entretien des lieux, enseignement, comptabilité, archivage...).

Pour autant, on ne saurait comprendre l'état présent du bouddhisme chinois en le prenant seulement à ses deux extrêmes, au temps de ses commencements, quand s'est dessiné le visage de la communauté monastique, et dans l'essor de la reconstruction des deux ou trois dernières décennies. Un mot doit être dit aussi des avatars qui ont marqué son histoire au cours des 150 dernières années. Car les destructions de la Révolution culturelle ont été précédées par celles qui ont marqué la rébellion des Taiping (1851-1864), notamment dans la Chine du sud, bastion bouddhiste traditionnel. L'effort subséquent de reconstruction coincidait alors avec la montée des critiques internes quant au système de formation et au (non-) respect effectif des préceptes. Le bouddhisme chinois entrait dans l'ère de l'aggiornamento. Certains des moines réformateurs prônaient essentiellement un retour aux disciplines antiques, sélectionnant un petit nombre de textes et de pratiques de méditation à privilégier. Un peu plus tard, un autre courant, dont le moine Taixu (1890-1947) est le représentant le plus célèbre, s'engagea dans une modernisation du bouddhisme, suivant une démarche proche de celle des républicains chinois du début du dernier siècle. Le rôle du laïcat était affirmé. L'éducation monastique devait aussi se rapprocher du mode d'enseignement dispensé par les universités occidentales. La création, dans cette première moitié du XX' siècle, de l'Association bouddhiste chinoise, la popularisation d'un "bouddhisme humaniste " ou " dans le monde " (renjian fojiao), les contacts alors entretenus entre moines et leaders politiques, toutes ces caractéristiques préfigurent déjà le visage qu'a pris le bouddhisme chinois après 1980. En même temps, les débats qui marquent la renaissance des années 1870-1940 se retrouvent encore aujourd'hui au sein d'une communauté bouddhiste qui doit désormais préciser ses rapports à la postmodernité d'une Chine en incessante transformation.

18. Erik Zürcher , Bouddhisme christianisme et société chinoise, Paris, Julliard, 1990, p. 26-27. 
Couverture d'un calendrier liturgique catholique de 2008. Il est écrit : « prions pour les jeux olympiques ».

(C) Taipei Ricci Institute

Le site de Wutaishan fournit un bon exemple de l'expansion bouddhiste contemporain comme des défis qui y sont attachés. Au milieu des années 1970, cette montagne, célèbre entre toutes dans l'histoire du bouddhisme chinois, ne comptait que quelques centaines de moines. Vers 2006, on en recensait environ 5000, date à laquelle ont été imposées quelques restrictions quant aux nombre de moines et moniales en résidence et en études sur le site. Des statistiques d'origine officielle donnent le chiffre de 2,8 millions de visiteurs s'étant rendus à Wutaishan en 2008, soit un revenu touristique associé de 1,4 milliard RMB. Le seuil des trois millions de visiteurs allait probablement être dépassé en $2009^{(19)}$. La taille et la vigueur des communautés en charge d'un tel ensemble, comme la masse des personnes que le site attire, structurent Wutaishan en centre de référence d'un nombre très important de communautés intermédiaires. Elles en font une force sociale au delà même de l'étroite délimitation de la sphère confessionnelle.

Les chiffres mentionnés à l'instant doivent cependant être interprétés avec prudence. Tout en retenant le chiffre de 9000 temples pour le seul bouddhisme han auxquels seraient rattachés 70000 moines, l'enquête de terrain poursuivie par Christian Cochini entre 2002 et 2006 dans 157 des plus grands monastères du pays montre par endroit des communautés monastiques encore fragiles ${ }^{(20)}$. La plupart des lieux recensés comptent entre 20 et 200 moines ou moniales en résidence, avec une moyenne oscillant entre 60 et 80 . Seuls quelques grands centres éducatifs (tel le temple Putou, siège de l'Institut d'études bouddhistes féminin de Wutaishan) dépassent ce nombre. La majorité des supérieurs de monastères sont nés entre 1955 et 1974, remplaçant souvent, à partir de 1995, une génération de moines nés durant les premières années de la République ou même juste avant. Ces supérieurs exercent à peu près toujours des responsabilités connexes dans l'Association bouddhique nationale ou ses relais provinciaux.

\section{Le catholicisme entre deux hétérodoxies}

En contraste avec celui du bouddhisme, le statut présent du catholicisme en Chine reste par bien des côtés celui d'une religion " aux marges. " La forte identité des villages chrétiens en faisait des entités souvent isolées du reste de leur environnement, l'équivalent d'un groupe ethnique minoritaire ("Catholicism as ethnicity" résume Richard Maden ${ }^{(21)}$ ). En ville, le réseau d'écoles et d'hôpitaux pouvait donner à l'Église une visibilité forte, mais elle structurait aussi des réseaux fonctionnant en marge de la société civile, voire de

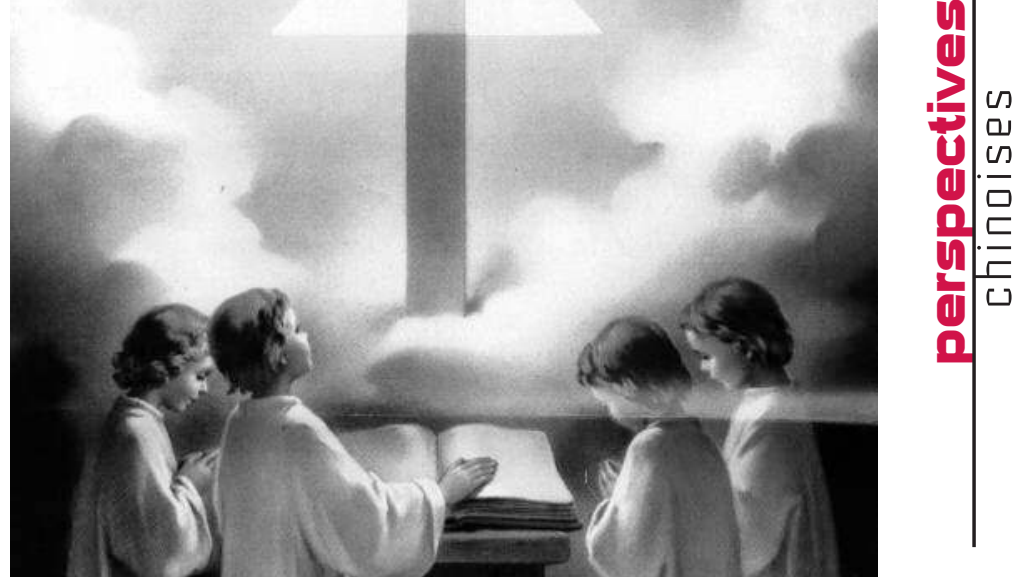

l'appareil d'État. Richard Madsen décrit du même coup le catholicisme chinois contemporain comme étant, par bien des côtés, l'équivalent d'une "religion hétérodoxe ${ }^{(22)}$ ". Le caractère de "religion populaire " que revêt le catholicisme dans les campagnes chinoises permet son enracinement culturel et social, tout en menaçant de l'aliéner " l'orthodoxie " catholique, le terme étant pris cette fois-là dans son sens ecclésial, en d'autres termes, le catholicisme chinois serait en danger permanent « d'hétérodoxie » tant du côté du pouvoir chinois que de celui de l'Église universelle.

Cette " hétérodoxie " de principe du catholicisme chinois provient aussi de son enracinement sur la longue durée : l'intensité des interactions culturelles intervenues entre missionnaires, lettrés et populations locales à partir de la fin du $\mathrm{XVI}$ e siècle s'est manifestée notamment dans les " espaces rituels » que constituent par exemple les funérailles et a abou-

19. Robert J. Saiget, dépêche AFP, 7 juillet 2009.

20. Christian Cochini, Guide des temples bouddhistes de Chine, Paris, Les Indes savantes, 2008.

21. R. Madsen, China's Catholics, Tragedy and Hope in an Emerging Civil Society, Berkeley, University of California Press, 1998, p. 54-56.

22. Cf. R. Madsen, "Beyond Orthodoxy: Catholicism as Chinese Folk Religion », in Stephen Uhalley Jr. et Xiaoxin Wu (éd.), China and Christianity: Burdened Past, Hopeful Future. Armonk, New York, M.E. Sharpe, 2001, p. 233-249. Les connotations entourant les expressions de xiejiao ou xiedao sont nombreuses en chinois. Le caractère xie renvoie à tout ce qui est pervers, déréglé, hétérodoxe, pernicieux. Dans le registre de la médecine chinoise il désigne des agents pathogènes, principalement exogènes. Même si les différentes écoles se sont souvent renvoyées le compliment, xiedao et xiejiao sont des épithètes que des confucéens ont d'abord réservés aux bouddhistes puis, à partir du XVIle siècle, à la doctrine chrétienne, l'« hétérodoxie » équivalant à peu près à la subversion de la tradition intellectuelle et du statu quo politique. 
ti à des synthèses culturelles que Nicolas Standaert a comparé aux produits évolutifs d'un " tissage " dans lesquels différents " fils " (textes employés, nature des objets rituels, pratiques musicales et dévotionnelles, calendrier respecté...) composent un texte symbolique en des synthèses dont la teneur dépend des temps et des agents ${ }^{(23)}$. En même temps, cette analyse permet de nuancer l'appréciation portée sur la marginalité des communautés catholiques : si elles sont dotées d'une forte identité propre, la manière dont leurs rituels ont évolué au cours du temps, souvent de manière inventive et complexe (la façon par exemple dont la célébration des funérailles mêle des éléments de la " religion diffuse " chinoise avec des pratiques exprimant les articles du Credo chrétien) permet d'établir des " ponts " avec d'autres communautés rituelles ${ }^{(24)}$. Les communautés catholiques chinoises sont distinctives, elles ne sont pas pour autant « étrangères » aux formes et sensibilités de la religiosité chinoise prise en sa transversalité.

Aujourd'hui, une analyse fondée sur un ensemble d'estimations parvient au chiffre d'une Église officielle groupant 5,3 millions de catholiques et d'une Église clandestine en comptant entre 12 et 14 millions. Le niveau de "service " offert varierait alors grandement, puisque l'Église officielle compterait environ 1900 prêtres, tandis que l'Église clandestine pourrait compter sur 1200. La différence serait plus nette encore pour le nombre de religieuses et de séminaristes. Le nombre des baptêmes déclarés par les diocèses semblait en nette augmentation en 2008 et 2009, notamment pour les diocèses touchés par le tremblement de terre de mai $2009^{(25)}$. La croissance ne doit pas dissimuler les crises latentes : l'avenir du catholicisme rural dépend de la résistance du type de sociabilité qui est associé à cette structure sociale. En ville, le catholicisme traditionnel souffre des mutations culturelles en cours. Mgr Aloysius Jin Luxian, évêque de Shanghai, évoquait dans une lettre pastorale de décembre $2007^{(26)}$ la faiblesse de la croissance numérique catholique comparée à celle des églises protestantes ${ }^{(27)}$, et en discernait les causes dans le manque d'esprit missionnaire des laïs. Ces derniers voient encore souvent le travail d'évangélisation comme le domaine d'action du clergé (prêtres et religieuses), quand ce même clergé, d'après l'évêque de Shanghai, dénonce le danger d'une dépendance vis-à-vis de la télévision et de l'Internet... Ce qui, à demimots, est ici en cause, c'est l'inadéquation du modèle paroissial classique dans une grande métropole contemporaine. Sans minorer ses spécificités, on peut estimer que le catholicisme chinois est et sera de plus en plus touché par la crise de gouvernance et de modèle sociétal que connait l'Église catholique dans son ensemble : le partage des pouvoirs et des responsabilités entre fidèles et clercs comme le statut de ces derniers, ou encore l'interprétation de la doctrine catholique entre tradition et modernité sont des débats qui influeront aussi sur la vitalité et la pertinence de l'Église chinoise.

\section{Le protestantisme, une religion chinoise en émergence?}

"On est tenté (observe Richard Madsen) de spéculer que, alors que le catholicisme correspond à une façon, pour les ruraux, de se protéger de la modernité, le protestantisme est un moyen pour eux de prendre force au moment d'entreprendre un dangereux pèlerinage dans la modernité ${ }^{(28)}$. "Le constat pêche par systématisme. Il n'en reste pas moins que le protestantisme chinois se caractérise par le sentiment d'empowerment, de forte confiance en soi, dont semble ordinairement jouir ses fidèles, comme par la vigueur de ses réseaux, lesquels transcendent largement les appartenances territoriales.

Les estimations du nombre de fidèles protestants en Chine varient entre 20 et 130 millions, les officiels chinois euxmêmes citant des chiffres discordants. Le champ est miné par la variété des groupes et des modes d'appartenance, dans une configuration qui, après tout, n'est pas sans rappeler celle des " nébuleuses " taoiste et bouddhiste, et qui fait alors du protestantisme une religion authentiquement chinoise... Il n'est pas évident pourtant qu'une estimation plus ou moins crédible soit hors de portée de l'enquêteur, et le recoupement des données disponibles apporte quelques lumières. Sur la base d'une enquête de terrain menée dans toutes les provinces sauf le Tibet, Werner Bürklin, de "China Partner " est parvenu en 2007 à une estimation de 39 millions de pro-

23. N. Standaert, The Interweawing of Rituals: Funerals in the cultural exchange between China and Europe, Seattle, University of Washington Press, 2008, p. 214-222.

24. Cf. ibid., p. 222-228.

25. China heute, 2009, 1 (161), p.7-10. Les statistiques de baptêmes (valables seulement pour l'Église officielle) sont compilées par l'hebdomadaire catholique Xinde (Foi), basé au Hebei, et restent incomplètes. La compilation des données de 90 diocèses en 2009 donne un chiffre de 22000 baptêmes entre janvier et avril 2009, soit une augmentation d'environ $14 \%$ par rapport à l'année précédente, avec une croissance particulièrement forte dans les diocèses de Chongqing et Chengdu. Xinde, 22 avril 2009, p. 1 ; dépêche Ucannews, 28 avril 2009.

26. Texte anglais disponible dans Tripod, vol. XXVIII, $n^{\circ} 149$, été 2008, p. 5-19.

27. Avant 1949, les églises protestantes comptaient environ 30000 fidèles et en dénombreraient en 2007 environ 200 000. Durant la même période, l'Église catholique serait passée de 100000 à 150000 (ibid., p.13). La lettre pastorale parle ici d'une "statistique incomplète ", mais ce chiffre comprend sans doute une partie des fidèles de l'Église clandestine. Le différentiel est donc moins net que ne l'indiquent les chiffres bruts, mais le dynamisme démographique supérieur des églises protestantes est établi.

28. " one is tempted to speculate that while Catholicism is a way for rural people to shelter themselves from modernity, Protestantism is a way to strengthen and fortify them as they undertake a dangerous pilgrimage into modernity ». R. Madsen, China's Catholics, op. cit., p.138. 
testants, divisés également entre les églises reconnues et non reconnues, un chiffre très proche de celui donné par l'enquête de l'Université normale de la Chine de l'Est déjà citée ${ }^{(29)}$. Même si le chiffre est une sous-estimation, comme l'affirment nombre d'associations évangéliques, il est fondé sur des données plus rigoureuses que les autres estimations. Le nombre total de protestants et catholiques en Chine serait donc compris entre 4 et $5 \%$ de la population totale.

Par ailleurs, la répartition des églises ne saurait s'effecteur sur l'axe d'une simple opposition entre les communautés locales rattachées au Mouvement des trois autonomies et celles qualifiées de clandestines. Don Snow a proposé de répartir les groupes locaux en cinq catégories ${ }^{(30)}$ : (a) les églises locales enregistrées auprès du Conseil chrétien chinois et le Mouvement patriotique des trois autonomies ; (b) les congrégations légalement enregistrées qui, néanmoins, ne se réunissent pas dans une église mais dans des maisons ou d'autres structures ; (c) les groupes qui gardent une dénomination d'origine étrangère (adventistes du septième jour...) ou se réclament d'une dénomination d'origine chinoise, groupes qui parfois célèbrent avec d'autres congrégations et parfois réussissent à faire enregistrer leurs lieux de culte auprès du bureau local des affaires religieuses ; (d) les " églises à la maison ", la plupart du temps situées en ville, qui ont fait le choix délibéré de ne pas être reconnues par les organisations protestantes patriotiques ; (e) les groupes ruraux, la plupart du temps non enregistrés, souvent d'orientation syncrétiste, et pourvus d'un leadership entreprenant. Si les estimations "prudentes " de la démographie protestante, comme celles utilisées dans cette étude, s'avèrent un jour être trop basses, ce sera par le fait d'une sous-estimation de la vitalité de cette dernière catégorie.

L'enquête de Werner Bürklin et les données fournies entre autres par Amity Foundation ${ }^{(31)}$ esquissent aussi une typologie régionale, plaçant les communautés protestantes les plus nombreuses dans le Henan, l'Anhui, le Zhejiang, le Fujian, le Guangdong, le Jiangsu, le Shanxi et le Shaanxi. Il faudrait pouvoir associer cette distribution géographique d'ensemble à une typologie des églises, en prêtant une attention spéciale aux principaux groupes non enregistrés tels que Les Crieurs (Huhanpai), le Chemin de la résurrection (Fuhuodao), la Communauté des disciples (Mentuhui). Le nombre, la variété et l'inventivité doctrinale de pareils groupes est sans nul doute un fait frappant du paysage religieux chinois d'ensemble. À de multiples égards, ces groupes peuvent apparaitre comme les successeurs des " cultes hétérodoxes" des siècles précédents. Une connaissance plus fine de leur enracinement régional permettrait sans doute de suggérer des filiations révélatrices, enracinant autrement le protestantisme contemporain dans l'histoire religieuse chinoise.

Si l'analyse ici esquissée était poursuivie pour s'étendre aux associations taoïstes, aux diverses manifestations de la religion populaire, aux rites et croyances des minorités nationales, aux religions ou para-religions nouvelles, sa conclusion n'en serait que renforcée : l'indéniable vitalité du paysage religieux chinois va de pair avec la multiplicité des formes communautaires et des degrés d'appartenance. Elle conjugue un "localisme " encore très marqué avec des tendances observables au niveau mondial ; les formes religieuses institutionnelles trouvent leur place dans la société chinoise tout en continuant à entretenir des rapports complexes, ondoyants et souvent malaisés avec les structures de l'État-Parti. Les enquêtes institutionnelles, démographiques et confessionnelles nous portent assez naturellement à passer d'une enquête sur " les religions chinoises " à la tentative d'interpréter " le champ religieux " dans sa globalité.

\section{Logiques du religieux}

Les spécificités du cas chinois ne signifient pas que, de principe, les catégories de la sociologie religieuse occidentale n'y soient pas applicables. Une nouvelle génération de sociologues chinois plaide même en faveur de leur pertinence, quitte à en infléchir ou enrichir leur usage ${ }^{(32)}$. Elle se distingue en cela de la génération de C.K. Yang, lequel appliquait aux religions chinoises davantage des catégories générales de la tradition sociologique que celles de la sociologie religieuse stricto sensu ${ }^{(33)}$. Il est vrai que, dans l'intervalle, tant les méthodes de la sociologie religieuse que le vécu religieux chinois ont considérablement évolué.

\section{Un marché religieux fragmenté}

Parmi ces tentatives, l'une notamment mérite que l'on s'y arrête : utilisant un schéma d'ensemble développé par Stark

29. Cf. www.assistnews.net, www.chinapartner.org consulté pour la dernière fois le 4 août 2009.

30. Dans une contribution d'abord publiée dans China News (Update), mars 2003, puis reproduite sur http://www.christianityinchina.org, consulté le 7 août 2009.

31. http://www.amitynewsservice.org, consulté le 7 août 2009.

32. Cf. Fenggang Yang, Joseph B. Tamney (éd.), State, Market, and Religions in Chinese Societies, Leiden, Brill, col. "Religion and the Social Order », 2005.

33. Si le nom de Joachim Wach apparaît en référence à la célèbre distinction entre religions diffuse et institutionnelle, l'emprunt initial renvoie bien à Talcott Parsons, comme C.K Yang lui-même l'indique dans sa brève préface. Cf. Religion in Chinese Society, Taipei, SMC Publishing, 1994 (1961), p.iii et 294. 
et Finke ${ }^{(34)}$, Fenggang Yang applique la théorie du « marché religieux » à la Chine contemporaine ${ }^{(35)}$, un marché religieux comprenant un ensemble d'activités, d'adhérents réels ou potentiels, d'organisations tentant de fidéliser et conquérir ces adhérents, et d'une culture religieuse offerte par ces organisations. Le marché d'un pays donné est affecté par le degré et les modes de régulation ou dérégulation du religieux mis en œuvre par l'État, l'interaction entre marché et normes de régulation créant une économie religieuse.

La thèse centrale de Yang est que le type de régulation mis en place par l'État chinois a conduit à la constitution de trois marchés religieux (ou à la fragmentation du marché religieux d'ensemble en trois marchés spécifiques), marchés qualifiés par lui de " rouge », « noir » et " gris ». Le marché rouge comprend les organisations, croyants et activités opérant sous l'approbation de l'État et obéissant à ses directives. Le marché noir est l'exact symétrique du premier, et opère donc sous le régime de l'illégalité. Le marché gris se définit d'abord et avant tout par l'ambiguité du cadre légal dans lequel il opère, et se divise en deux sous-ensembles : (a) les activités illégales des organisations légalement reconnues (évangélisation à la maison ou sous couvert de service social conduit par des groupes placés sous la tutelle des associations patriotiques protestantes ou catholiques par exemple) ; (b) les activités religieuses qui se tiennent sous un autre couvert, par exemple scientifique ou culturel (quasi-religions, pratiques " folkloriques ", populaires ou " de santé ", reconstruction des temples et renaissance de formes religieuses traditionnelles dans le sud-est du pays....). Les restrictions du marché rouge et les dangers associés au marché noir provoquent inévitablement, poursuit Yang, la croissance du marché gris. Il en voit des prémices dans le culte rendu à Mao durant la révolution culturelle, puis une première expression dans la fièvre du qigong ${ }^{(36)}$. Yang se risque même à des estimations, comptant environ 100 millions de participants au marché religieux rouge, 200 millions au marché noir, et estimant à " plusieurs centaines de millions " le nombre d'adhérents potentiels du marché gris, ces personnes constituant des cibles privilégiées pour le renouveau de la religion diffuse et plus encore sans doute pour les nouveaux mouvements religieux si ces derniers pouvaient trouver une expression publique $^{(37)}$.

L'analyse que mène Yang de "l'économie religieuse chinoise " souffre d'être réduite aux seules dimensions d'acteurs individuels d'une part, de l'État d'autre part. Elle pâtit aussi de l'extrême élasticité de sa définition du " marché gris ". Mais elle rend bien compte, par exemple, des stratégies individuelles des adhérents de Falungong et autres membres des groupes de qigong après 1999 : passage du qigong au marché noir pour les pratiquants prêts à payer le prix de leur détermination ; retrait du marché religieux pour d'autres ; entrée sur le marché rouge ou gris du christianisme ou du bouddhisme pour d'autres encore ; et enfin reconstruction lente et prudente d'un nouveau segment de " marché gris " au travers de la reconnaissance des associations qigong de santé (jianshen qigong). Cette approche en termes d'économie religieuse permet aussi de conceptualiser le paradoxe chinois : d'une part, la Chine connait une grande fragmentation des expressions religieuses, fragmentation causée par le mode de régulation imposé par l'État; mais d'un autre côté, il faut savoir appréhender le fait religieux chinois dans sa continuité, les choix effectués par les individus s'avérant (a) mouvants dans leur expression en fonction du prix qu'ils doivent payer pour satisfaire leur demande latente selon l'évolution des régulations et de leur application ; (b) constants en termes de "besoins exprimés ", même si le prix imposé par l'État module le niveau et le mode de consommation religieuse ${ }^{(38)}$.

Poursuivant l'analyse, il faudrait se demander si le marché religieux n'est pas lui-même inclus dans un "marché du sens " plus large, dont le nationalisme, les " études nationales " (guoxue), le sport et l'art constitueraient d'autres expressions, chacune en concurrence avec les autres et justiciables d'une analyse en termes de coût à payer rapporté à la satisfaction escomptée, la satisfaction en question étant définie par la conviction de mener une vie sensée sur le plan personnel et rapportée à la destinée d'un groupe (collectivité naturelle, nation, église, humanité...) ${ }^{(39)}$. La prétention étatique de contrôler le "marché du sens " par le biais de la " civilisation spirituelle " (jingshen wenming) a finalement contribué à l'exaspération croissante de la demande,

34. Stark, Rodney and Roger Finke. Acts of Faith: Explaining the Human Side of Religion Berkeley

and Los Angeles: University of California Press, 2000

35. Fenggang Yang, "The Red, Black, and Gray Markets of Religion in China ", The Sociological Quarterly , 47 (2006), p. 93-122. L'auteur a fait paraitre une version chinoise du même article ; « Zhongguo zongjiao de san se shichang », Zhongguo renmin daxue bao (Journal of Renmin University of China), 2006 (6), p.41-47.

36. Ibid., p. 98-99.

37. Ibid., p. 113-114.

38. Yang ne se pose pas la question d'une augmentation possible de la « demande globale » stimulée par la satisfaction des besoins matériels, ni celle d'une fluctuation de la demande en fonction de cycles privé public similaires à ceux théorisés par A. 0 Hirschman in Shifting Involvements, Private Interest and Public Action, Princeton U.P., 1982

39. Paul Tillich a été l'un des premiers à thématiser l'inclusion des religions historiques dans une sphère du sens que la modernité élargit aux « quasi-religions séculières ». La « religion » dans son acception la plus étendue correspond alors à « l'état d'être saisi par une préoccupation ultime » qui répond "à la question du sens de notre vie ». Cf. Le Christianisme et les Religions, Paris, Aubier-Montaigne, 1968, p. 65-68. 
confrontée à une offre inadéquate. En même temps, les réponses proprement religieuses à cette demande sociale peuvent s'avérer trop coûteuses pour certains acteurs, ou ne pas répondre pleinement à la nature de l'attente qu'ils expriment, ou provoquer à terme la déception de certains, ou encore perdre en valeur relative face à la concurrence de nouveaux vecteurs d'offre, la vogue des " études nationales » représentant un cas d'espèce.

\section{Vers une économie religieuse intégrée}

La question qui surgit à ce stade est celle de savoir si le paradigme du marché rend compte de façon adéquate des phénomènes fondamentaux qui déterminent le " religieux " en Chine, ou si d'autres paradigmes ne doivent pas s'y substituer partiellement ou totalement. Au reste, la plupart des études ethnologiques ne font pas de l'acteur individuel l'agent premier du marché religieux chinois pris dans sa globalité, que ce marché soit ou non surdéterminé par les régulations étatiques. Les comportements et les choix des acteurs individuels sont ordinairement rapportés à des sphères mutuellement corrélées. Curieusement, c'est l'attention portée à la structure traditionnelle des " marchés " locaux, à leur mise en réseau, à la résilience de communautés commerciales et d'alliances culturellement endogènes ${ }^{(40)}$, c'est cette attention aux cadres spatiaux de l'activité économique donc, qui a donné lieu à la mise au jour de "sphères de croyance ", souvent identifiées à des sphères d'alliance matrimoniales et à des "sphères rituelles" (jisi quan) ${ }^{(41)}$. Ces dernières s'avèrent difficiles à décrire lorsqu'elles incluent des lieux de pèlerinages parfois très éloignés du network local. Dans l'approche de Skinner, la "sphère » dépasse par définition le cadre du village. Mais, dans l'utilisation plus lâche qui en a été faite par la suite, le terme est souvent appliqué à toute unité cultuelle qui s'avère structurée et pertinente, $y$ compris (éventuellement) le village ${ }^{(42)}$. Néanmoins, il s'applique avec plus de justesse à un nexus, croisant alors un ensemble de communautés selon un système parfois très complexe d'alliances et d'intérêts. Même dans ce cas, la sphère est d'abord spatiale, dans le sens où elle construit les frontières d'un territoire symbolique, délimité et souvent protégé par l'objet et la forme des pratiques rituelles.

À première vue, la description de ces sphères induit l'appréhension du religieux en termes qui laissent peu de place au paradigme du marché, non pas tant parce que l'individu ne tient dans la sphère qu'un rôle subordonné que parce que l'éventail des choix d'appartenance, croyance et pratique religieuses est fortement restreint et encadré. Si bien que l'inventivité religieuse et l'adaptation aux évolutions globales se manifestent moins par le remplacement d'un " produit " par un autre que par une logique d'adaptation interne qui fait parfois évoluer très profondément l'offre religieuse disponible sans en changer la configuration originelle. C'est une économie corporatiste du religieux que décrivent les constructions en termes de "sphères" ou de " champ ". Et cependant, il nous faut noter que l'attention aux "sphères de croyances " peut rendre compte de phénomènes de conversion, pour autant qu'attention soit prêtée à leur caractère collectif et à la manière dont la conversion renforce et redynamise la sphère locale, laquelle, en effectuant pareil saut, retravaille ses marqueurs identitaires et ses repères symboliques plutôt qu'elle ne les abandonne. La diversité des formes de reconstruction de la vie religieuse villageoise après la Révolution culturelle, comme la décrit par exemple Thomas DuBois pour un comté du Hebei ${ }^{(43)}$, est une illustration du phénomène : le village peut connaitre une vie religieuse intense ou limitée, il peut entretenir un temple de village ou être centré autour d'un groupe religieux dominant héritier des cultes "hétérodoxes " des siècles précédents. Dans tous les cas (conversion d'une religion à une autre, mode de reconstruction de l'identité religieuse du village par réinterprétation du système ancien), un choix religieux est effectué, mais qui semble être le fait d'un acteur collectif plutôt que la résultante de choix individuels. C'est le mode d'interaction en vigueur dans la communauté villageoise et la nature des besoins qu' elle tente de satisfaire $^{(44)}$ qui s'avèrent alors les facteurs décisifs de la reconfiguration religieuse.

40. W. Skinner, " Marketing and Social Structure in Rural China ». Journal of Asian Studies 24(1): 3-43, 24(2): 195-228, 1964

41. Cf. Par exemple Lin Mei-jung, «La sphère rituelle comme forme d'organisation locale : le cas de l'agglomération de Tsaotun " (you jisi quan lai kan caodun zhen de difang zhzhi), Bulletin of the Institute of Ethnology, Taipei, Academia Sinica, $\mathrm{n}^{\circ} 62$, automne 1986, p. 53-114.

42. Voir les études classiques de Stephan Sangren, History and Power in a Chinese Community, Stanford, Stanford University Press, 1987; David Faure, The structure of Chinese Rural Society: Lineage and Village in the Eastern New Territories, Hong Kong, Hong Kong and Oxford University Press, 1986. Parmi d'autres, Burton Pasternak a montré que le rituel célébré au niveau du village pouvait être ou ne pas être facteur de cohésion communautaire, et que de nombreux facteurs déterminaient la place et l'importance de ces activités rituelles proprement locales (cf. Kinship and Community in Two Chinese Villages, Stanfrod, Stanford U.P., 1972, notamment p. 109-113 et 125-127). En d'autres termes, la première question que se pose l'ethnographe est celle de l'extension, de la pertinence et de la corrélation des sphères rituelles ou croyantes, sans présupposer la primauté de principe de telle ou telle dimension territoriale.

43. The Sacred Village: Social Change and Religious Life in Rural North China, Honolulu, University of Hawai'i Press, 2005, p. 39 sq.

44. Une fois encore ces besoins peuvent être divers : une faible pluviométrie, la place accordée aux lignages, la situation sanitaire locale ou encore l'intensité et la prévalence des conflits peuvent peser lourdement sur la hiérarchisation des besoins symboliques. 
On pourrait en fin de compte proposer le schéma suivant : les acteurs collectifs que sont le village, la guilde, l'association pieuse structurent un premier " marché du religieux " qui fut longtemps le marché dominant - marché un temps supprimé et qui tente aujourd'hui de trouver sa place propre. Ces acteurs négociaient le prix des intervenants religieux ${ }^{(45)}$ et laissaient peu de place à un marché d'acteurs individuels. Il existait une certaine élasticité des formes et du coût des prestations religieuses à l'intérieur du segment de marché dans lequel ils opéraient mais, à l'inverse, le changement d'une forme religieuse à une autre (conversion d'un village au christianisme par exemple) s'effectuait à un prix très lourd et, de toute façon, l'offre alternative à l'existant était pauvre. Cependant, il a toujours existé un second marché religieux activé par les individus (ou par des familles indépendamment de la logique villageoise ou lignagère) : l'affiliation à un "culte hétérodoxe " par exemple s'effectuait en grande partie sur une base individuelle ${ }^{(4)}$; la " sortie du monde " (chu jia) était un choix alternatif, les migrations s'accompagnaient et s'accompagnent de mutation des affiliations religieuses... "L'économie religieuse " chinoise fonctionnait (et d'une certaine façon fonctionne toujours) par le jeu des multiples formes d'interaction entre trois acteurs : l'État, à la fois régulateur du marché et possesseur lui-même d'une " offre " religieuse ou quasi religieuse ; un ensemble d'acteurs collectifs régulateurs des rituels; et des acteurs individuels susceptibles de déserter un segment donné du marché et d'en organiser un autre. L'individualisation des acteurs, affirment souvent les chercheurs chinois, est bien aujourd'hui le fait le plus marquant. Il va de pair, ajoutentils, avec l'entrée des activités religieuses dans les mécanismes du marché, la modernisation des croyances et attitudes religieuses, et enfin une compétition pacifique entre les diverses croyances et organisations ${ }^{(47)}$.

\section{Une * sortie de la religion* aux caractéristiques chinoises}

C'est à ce stade qu'il nous est possible de revenir sur l'usage même du terme "religieux ", et de nous redemander s'il ne plaque pas sur les réalités étudiées des catégories préfabriquées qui brident la compréhension plus qu'elles ne l'éclairent.

Les sociétés où l'État a pris forme sont celles-là même où s'est effectué la séparation de la sphère politico-étatique de celle du religieux. Telle est du moins la thèse célèbre de Marcel Gauchet, dont il n'est pas question de discuter ici tous les présupposés ni toutes les implications. Quelques rappels peuvent néanmoins aider à une nouvelle intelligibilité de la structuration du religieux en Chine. Si l'on comprend par le terme de religion l'opération par laquelle un groupe humain rapporte son existence à un fondement extérieur à lui-même et sur lequel il n'a d'autre prise que celle que lui assurent le rite ou la magie (ou encore, comme le dit Gauchet, "la relation de l'humanité avec elle-même sous le signe de la dépossession ${ }^{(4)}$ "), la naissance du politique (partout où elle n'est pas interdite par les choix virtuels effectués à l'intérieur des "sociétés sans État ") opère par étapes la spécification et la limitation de la sphère religieuse en instaurant une série de médiations de la société avec elle-même. Avec l'apparition de l'État "s'opère une matérialisation de l'extériorité religieuse dans l'espace collectif " ${ }^{(4)}$. Plus tard, avec la formation de l'État moderne (" l'État en possession de son concept "), " en se séparant du religieux, le politique apparait au grand jour et devient identifiable en lui-même et pour lui-même ${ }^{(50)}$ ".

Dans cette perspective "la révolution de la sortie de la religion ", est celle de "la condition politique assumée en conscience $^{(51)}$ ". En d'autres termes, la " sortie de la religion " correspond à la situation d'un groupe qui se comprend comme communauté capable de déterminer son mode d'existence et son destin selon les seules médiations imposées par les règles de la discussion politique, sans que nul référent sacral n'en contraigne le cours. Bien certainement, en Chine, le surgissement de la condition politique ne s'est pas effectué selon les canaux et la temporalité qui furent celles de l'Occident. Cette divergence est concomitante de la divergence observée dans la structuration et les évolutions de la sphère religieuse. Mais l'apparition, même au titre de virtualité, de la condition politique ne peut pas ne pas provoquer la marche vers une "spécialisation " religieuse jusqu'à la perte ultime de l'efficacité sociale de la sphère religieuse en tant que fondatrice du social même ${ }^{(52)}$. Si l'apparition précoce de l'État chinois a très tôt

45. Sur la variation de la rémunération des intermédiaires religieux en fonction de l'offre et de la demande, et son élasticité mitigée en fonction de la communauté de référence, voir les exemples fournis par Donald S. Sutton, "Transmission in Popular Religion ; The Jiajiang Festival Troupe of Southern Taiwan ", in Meir Shahar et Robert P. Weller (éd.), Unruly Gods : Divinity and Society in China, Honolulu, University of Hawai'i Preess, 1996, p. 220-221 et par Thomas DuBois, The Sacred Village : Social Change and Religious Life in Rural North China, op. cit., p. 61 sq..

46. Cf. l'historique de l'implantation de la Secte $L i$ à Tianjin durant les années 1930 in Thomas DuBois, The Sacred Village , op. cit., p.113-121.

47. Cf. par exemple Hu Changshen « 21 sheji zhongguo zongjiao de yanbian qushi xintan (nouvelles recherches sur les tendances et transformations des religions en Chine au XXle siècle) », Zhonghua wenhua Iuntan (Forum de la culture chinoise), 2002, 1, p. 3134.

48. Marcel Gauchet, La condition politique, Paris, Gallimard, col. « Tel », 2005, p. 14.

49. Ibid., p.17.

50. Ibid., p.19.

51. Ibid., p.20 
subordonné l'expression sociale de la fonction religieuse aux formes politiques propres à la Chine, ces mêmes formes politiques auront très longtemps préservé le fondement sacral de l'être-ensemble social. Dans cette optique, il n'est sans doute pas exact de décrire le tournant intervenu à la fin du XIX siècle comme une " rupture " qui aurait artificiellement fait basculer le "religieux " chinois dans les " religions » à l'occidentale, bouleversant l'éthos du premier. Plutôt, toutes les étapes historiques qui ont précédé ce moment (émergence, parmi les ruines de l'empire han, du taoisme et du bouddhisme comme religions constituées, "spécialisées ", ruptures des solidarités traditionnelles induites par la formation périodique de " cultes hétérodoxes ", reformulations confucianistes, Querelle des Rites, cristallisations anticléricales...) peuvent être lues en continuité. Dans l'histoire longue, la Chine a bien connu une structuration progressive de la sphère du religieux signant la distinction entre la sphère du religieux et les autres domaines de l'activité sociale. Non seulement les religions chinoises sont un objet historique en perpétuelle évolution, redéfinition et autonomisation, mais encore leur involution les confronte aujourd'hui à la question de la " sortie du religieux " en son expression globale.

Pour le dire autrement : dans l'histoire longue, l'État chinois a mobilisé et perpétuellement reformulé les ressources religieuses existantes de façon à fonder et pérenniser tant les représentations que le corps social se donne de lui-même que le mode de contrôle qu'il exerçait sur lui. Ce fait massif ne doit pas nous empêcher de lire de façon diachronique l'émergence de formes religieuses autonomes et de débats d'idées propres à la Chine qui ont peu à peu affaibli le lien politicoreligieux compris comme fondement sacral du social. D'une façon analogue à celle observable dans d'autres contextes sociaux, l'affaiblissement d'un pareil lien travaille dans le sens d'une entrée de la communauté nationale chinoise dans l'ère de la " condition politique ", à lire, dans le même mouvement, comme une "sortie du religieux ". Un processus loin d'être achevé, peut-être même inachevable par essence. Le simple fait d'identifier pareille logique n'en reste pas moins important pour se délivrer de l'illusion qui consiste à interpréter les évolutions religieuses chinoises sur le seul mode de " ruptures externes » intervenues au cours des 60 , des 100 ou des 150 dernières années.

\section{Le confucianisme contemporain, vraie religion de la sortie de la religion?}

Un exemple de ce qui est ici en jeu est fourni par le confucianisme contemporain. Vers la fin du XIX ${ }^{\mathrm{e}}$ siècle, la géné- ralisation des savoirs occidentaux, des catégories épistémologiques (science, religion, politique) et des institutions sociales (universités, églises, presse et partis) qui leur sont associés remet en cause l'autoreprésentation du confucianisme comme système rituel, sapiental et cosmologique englobant et, du même coup, l'ordre culturel et social de la Chine ${ }^{(53)}$. Aux tentatives de sauver l'universalité de l'enseignement (jiao) confucéen succèderont ses reformulations républicaines, soit comme une religion (conçue en rapport et contraste avec le christianisme, modèle épistémique de la religion), soit comme philosophie, là encore dans un rapport de tension créatrice avec la tradition occidentale. Après la tourmente maoiste, la « reconstruction " confucéenne - parallèle à et concurrente de la reconstruction religieuse - passe par différentes étapes et stratégies. Des chercheurs redécouvrent la vitalité d'un " confucianisme populaire " organisé autour des temples lignages (citang), créateur de continuité et d'harmonie communautaire. L'essor de la Chine et des petits dragons suscite des débats animés autour de l'efficacité socio-économique de la sagesse confucéenne ; l'affirmation nationale voire nationaliste se traduit en un corpus toujours grandissant de réinterprétations du canon confucéen, réinterprétations qui s'adressent à des publics très variés mais visent une frange toujours plus large de la population chinoise. En corolaire, ressurgit la question de la " nature religieuse " du confucianisme, et nombre de systèmes fleurissent qui entendent en expliciter la nature ultime, la fonction sociale, et son inscription souhaitable dans les structures légales. L'essentiel, dans ce mouvement de fond de reconstruction sociale et intellectuelle, c'est qu'il s'offre en alternative aux catégories occidentales qui avaient triomphé à la fin de l'Empire Qing, et qu'il questionne les frontières établies entre science, philosophie, sagesse et religion ${ }^{(54)}$, le monopole de l'université sur la dispensation du savoir et la fonctionnalisation d'un espace politique conçu indépendamment de la tradition nationale qui l'habite.

Ces tentatives encore dispersées et inchoatives entendent donc tout à la fois affirmer la valeur religieuse du confucianisme et contester la façon dont l'Occident a imposé sur l'ancien ordre social et cosmologique chinois son propre découpage entre les différents plans de l'existence et de la connaissance. Une reformulation contemporaine dont il est

52. Ce que réalise précisément le caractère « diffus » du religieux chinois.

53. Cette synthèse est inspirée de Sébatien Billoud et Joël Thoraval, « Anshen liming ou la dimension religieuse du confucianisme », Perspectives chinoises, $n^{\circ} 3,2008$, p. 96-116.

54. Quitte à emprunter à des reconstructions épistémologiques occidentales, telles celles d'llya Prigogine. Cf. S. Billoud et J. Thoraval, « Anshen liming ou la dimension religieuse du confucianisme », art. cit., p. 108. 
assez clair qu' elle ne saurait conduire à la reviviscence d'un passé révolu et largement imaginé, mais dont il faut se demander si elle n'érige pas le confucianisme en religion chinoise de la sortie de la religion.

Religion chinoise, dans la mesure où le confucianisme entend bien ancrer le lien social dans une transcendance ${ }^{(5)}$. Cette transcendance est inscrite dans une interprétation de l'histoire nationale et culturelle chinoise qui conjugue la spécificité de son apport humaniste et la revendication d'une connaissance de type universel sur la nature de l'homme et ses capacités d'autodépassement. Mais religion de la sortie de la religion dans la mesure où le modèle confucéen de la connaissance et de l'action conteste, d'une part les distinctions établies par l'Occident (et même par le monde indien) entre sciences, religion et autres domaines de la pratique sociale, d'autre part les formes et le sens des rituels communément qualifiés de « religieux ». C'est donc la synthèse déjà suggérée en son temps par Confucius (critique du religieux jointe à un accommodement avec ce dernier), synthèse reformulée après la rencontre intellectuelle avec l'Occident, qui paradoxalement projette le confucianisme dans l'ère de la postmodernité. Dans cette logique, alors que la tradition chrétienne aurait été amenée à accomplir un long et douloureux cheminement au travers de ses formes et de son essence pour " sortir de la religion », le confucianisme dépasserait dès son principe l'étape historique $\mathrm{du}$ " religieux au fondement du lien social »- et ce, tout en fournissant les ressources de sens permettant de maintenir un fondement quasi sacral $\mathrm{du}$ lien social - fondement que l'Occident postmoderne n'est plus en mesure d'assurer.

\section{L'instrumentalisation des religions}

Un autre exemple de la "sortie de la religion " en régime chinois est paradoxalement fourni par les appels qu'adressent aux religions gouvernants et gouvernés. L'absence désormais totale d'un fondement transcendant du social (fondement que pouvait encore prétendre fournir le maois$\mathrm{me}$ ) érige les religions en dispensatrices auxiliaires d'une " harmonie » qu' elles ne peuvent plus assurer par leurs rituels et leur enracinement social. $\grave{A}$ défaut, les religions " fabriquent " de l'harmonie sur le mode d'un " construit " théorique, c'est-à-dire d'une réinterprétation de leurs traditions guidée par les impératifs d'un pouvoir soucieux de gérer au mieux les transformations sociales en cours. Ainsi, certains chercheurs ont suggéré qu'il n'était pas absurde de parler d'une " conversation " dans la longue durée entre les leaders protestants et l'État-Parti ${ }^{\left({ }^{6}\right)}$ : la préoccupation des Églises protestantes, dès l'ère républicaine, de participer à la construction d'un État moderne, la personnalité et l'influence de l'évêque $\mathrm{K}$. H. Ting ${ }^{(57)}$, l'examen des négociations quant au mode de gestion des affaires religieuses intervenues après 1980, la construction théologique poursuivie autour des notions d'harmonie ou de socialisme montrent de fait une intensité d'interaction qui participe aussi de la modernisation étatique chinoise. En même temps, c'est justement cette intensité qui a exacerbé les divisions à l'intérieur du protestantisme chinois. C'est là un exemple de la réinterprétation et de la sécularisation des doctrines religieuses en fonction des impératifs nationaux, et une analyse de ce type pourrait par exemple être menée sur les constructions du renjian fojiao (bouddhisme dans le monde) en tant qu'elles fournissent un mode de civilité qui atteste de la pertinence sociale de la tradition ainsi réinterprétée.

Ainsi, les formes que prend le réveil religieux chinois traduisent souvent une nostalgie du fondement politico-religieux du lien social - les religions étant aujourd'hui susceptibles d'être instrumentalisées justement parce que le religieux ne fournit plus l'ancrage sacral du vivre-ensemble. De ce fait même, on peut interpréter le réveil religieux de la Chine dans les termes d'une transition historique : celle de l'hésitation de ce pays au seuil d'une autonomisation du politique, tant la tentation de l'ancrage du lien social dans un référent sacral demeure forte.

Dans un contexte marqué tout à la fois par un contrôle étatique maintenu et la perte de la maitrise par l'État des formes et des catégories du religieux, le bourgeonnement religieux est tout à la fois possible prélude à et substitut présent de la démocratisation (ou en tout cas de l'autonomisation de la société civile). L'instrumentalisation croissante des religions chinoises va de pair avec la perte de tout fondement religieux du lien social, inscrivant la Chine dans la sortie du religieux aussi inachevée et tourmentée que se révèle être pareille sortie. Parmi d'autres expressions religieuses, protestantisme et bouddhisme ambitionnent aujourd'hui l'un et l'autre d'être les expressions tout à la fois locales et globales d'un religieux qui n'est plus fondateur mais supplétif du lien

55. La question de la nature religieuse du confucianisme a donné lieu à d'innombrables débats. Pour un aperçu de la question depuis 1978 on pourra consulter John Makeham, Lost Soul : Confucianism in Contemporary Chinese Academic Discourses, Cambridge, Harvard University Asia Center, 2008.

56. Cf. Ryan Dunch, " Christianity and "Adaptation to Socialism" ", in Mayfair Mei-hui Yang (éd.), Chinese Religiosities, Afflictions of Modernity and State Formation, Berkeley, University of California Press, 2008, p. 155-178.

57. Sur la figure centrale de K. H. Ting, voir l'étude très compète de Philip L. Wickeri, Reconstructing Christianity in China : K. H. Ting and the Chinese Church, Maryknoll, N. Y., Orbis Books, 2007. 
social. Parallèlement, un confucianisme recomposé aspire pour sa part à devenir la forme religieuse achevée de la sortie de la religion. On le voit : l'étude des mutations religieuses que connait aujourd'hui la Chine ne revêt pas un intérêt simplement ethnographique. Rapportée à d'autres situations, elle fournit un apport précieux à l'étude des recompositions du vivre-ensemble dans des sociétés confrontées à l'émergence d'une communauté globale dont le fondement n'est autre que le destin qu'elle se fixera à elle-même. •

\section{Glossaire}

chujia 出家 citang 祠堂 fang 放 fensanxing 分散性 fuhuodao 復活道 guiyi 的依 guoxue 國學

hanyu shenxue 漢語神學 hehe 和合 hehexue 和合學 hexie 和諧 huhanpai 呼喊派

jianshen qigong 健身氣功 jiao 教 jingsheng wenming 精神文明 jisi quan 祭祀圈 jushi 居士 kuosanxing 擴散性 lixue 理學 mentuhui 門徒會 ming 命 misanxing 彌散性 qishi 啟示 renjian fojiao 人間佛教 sankaide 散開的 shangdi yesu 上帝耶穌 sheng sheng bu xi 生生不息 shou 收 wu 悟 xiedao 邪道 xiejiao 邪教 xinxing zongjiao 新興宗教 yiguandao 一貫道 yun 運 zhihui 智慧 zhizhe 智者 